\title{
Diagnostic criteria and severity assessment of acute cholangitis: Tokyo Guidelines
}

\author{
Keita Wada ${ }^{1}$, Tadahiro Takada ${ }^{1}$, Yoshifumi Kawarada ${ }^{2}$, Yuji Nimura ${ }^{3}$, Fumihiko Miura ${ }^{1}$, \\ Masahiro Yoshida $^{1}$, Toshihiko Mayumi ${ }^{4}$, Steven Strasberg ${ }^{5}$, Henry A. Pitt ${ }^{6}$, Thomas R. Gadacz ${ }^{7}$, \\ Markus W. Büchler ${ }^{8}$, Jacques Belghiti ${ }^{9}$, Eduardo de Santibanes ${ }^{10}$, Dirk J. Gouma ${ }^{11}$, Horst Neuhaus ${ }^{12}$, \\ Christos Dervenis ${ }^{13}$, Sheung-Tat Fan ${ }^{14}$, Mirn-Fu Chen ${ }^{15}$, Chen-Guo Ker ${ }^{16}$, Philippus C. Bornman ${ }^{17}$, \\ Serafin C. Hilvano ${ }^{18}$, Sun-Whe Kim ${ }^{19}$, Kui-Hin Liau ${ }^{20}$, and Myung-Hwan Kim ${ }^{21}$ \\ ${ }^{1}$ Department of Surgery, Teikyo University School of Medicine, 2-11-1 Kaga, Itabashi-ku, Tokyo 173-8605, Japan \\ ${ }^{2}$ Mie University School of Medicine, Mie, Japan \\ ${ }^{3}$ Division of Surgical Oncology, Department of Surgery, Nagoya University Graduate School of Medicine, Nagoya, Japan \\ ${ }^{4}$ Department of Emergency Medicine and Intensive Care, Nagoya University School of Medicine, Nagoya, Japan \\ ${ }^{5}$ Department of Surgery, Washington University in St Louis and Barnes-Jewish Hospital, St Louis, USA \\ ${ }^{6}$ Department of Surgery, Indiana University School of Medicine, Indianapolis, USA \\ ${ }^{7}$ Department of Gastrointestinal Surgery, Medical College of Georgia, Georgia, USA \\ ${ }^{8}$ Department of Surgery, University of Heidelberg, Heidelberg, Germany \\ ${ }^{9}$ Department of Digestive Surgery and Transplantation, Hospital Beaujon, Clichy, France \\ ${ }^{10}$ Department of Surgery, University of Buenos Aires, Buenos Aires, Argentina \\ ${ }^{11}$ Department of Surgery, Academic Medical Center, Amsterdam, The Netherlands \\ ${ }^{12}$ Department of Internal Medicine, Evangelisches Krankenhaus Düsseldorf, Düsseldorf, Germany \\ ${ }^{13}$ First Department of Surgery, Agia Olga Hospital, Athens, Greece \\ ${ }^{14}$ Department of Surgery, The University of Hong Kong, Hong Kong, China \\ ${ }^{15}$ Department of Surgery, Chang Gung Memorial Hospital, Chang Gung University, Taoyuan, Taiwan \\ ${ }^{16}$ Division of HPB Surgery, Yuan's General Hospital, Taoyuan, Taiwan \\ ${ }^{17}$ Division of General Surgery, University of Cape Town, Cape Town, South Africa \\ ${ }^{18}$ Department of Surgery, Philippine General Hospital, University of the Philippines, Manila, Philippines \\ ${ }^{19}$ Department of Surgery, Seoul National University College of Medicine, Seoul, Korea \\ ${ }^{20}$ Department of Surgery, Tan Tock Seng Hospital/Hepatobiliary Surgery, Medical Centre, Singapore \\ ${ }^{21}$ Department of Internal Medicine, Asan Medical Center, University of Ulsan, Seoul, Korea
}

\begin{abstract}
Because acute cholangitis sometimes rapidly progresses to a severe form accompanied by organ dysfunction, caused by the systemic inflammatory response syndrome (SIRS) and/or sepsis, prompt diagnosis and severity assessment are necessary for appropriate management, including intensive care with organ support and urgent biliary drainage in addition to medical treatment. However, because there have been no standard criteria for the diagnosis and severity assessment of acute cholangitis, practical clinical guidelines have never been established. The aim of this part of the Tokyo Guidelines is to propose new criteria for the diagnosis and severity assessment of acute cholangitis based on a systematic review of the literature and the consensus of experts reached at the International Consensus Meeting held in Tokyo 2006. Acute cholangitis can be diagnosed if the clinical manifestations of Charcot's triad, i.e., fever and/or chills, abdominal pain (right upper quadrant or epigastric), and jaundice are present. When not all of the components of the triad are present, then a definite diagnosis can be made if laboratory data and imaging findings supporting the evidence of inflammation and biliary obstruction are obtained. The severity of acute cholangitis can be classified into three grades, mild (grade I), moderate (grade II), and
\end{abstract}

Offprint requests to: $\mathrm{K}$. Wada

Received: May 31, 2006 / Accepted: August 6, 2006 severe (grade III), on the basis of two clinical factors, the onset of organ dysfunction and the response to the initial medical treatment. "Severe (grade III)" acute cholangitis is defined as acute cholangitis accompanied by at least one new-onset organ dysfunction. "Moderate (grade II)" acute cholangitis is defined as acute cholangitis that is unaccompanied by organ dysfunction, but that does not respond to the initial medical treatment, with the clinical manifestations and/or laboratory data not improved. "Mild (grade I)" acute cholangitis is defined as acute cholangitis that responds to the initial medical treatment, with the clinical findings improved.

Key words Cholangitis · Diagnosis · Severity of illness index · Guidelines

\section{Introduction}

The pathogenesis of acute cholangitis is biliary infection associated with partial or complete obstruction of the biliary system caused by any of various etiologies including choledocholithiasis, benign and malignant strictures, biliary-enteric anastomotic malfunction, and indwelling biliary stent malfunction. Biliary infection alone does not cause clinical cholangitis unless biliary 
obstruction raises the intraductal pressure in the bile duct to levels high enough to cause cholangiovenous or cholangiolymphatic reflux. ${ }^{1}$ Thus, acute cholangitis progresses from local biliary infection to the systemic inflammatory response syndrome (SIRS), and advanced disease leads to sepsis with or without organ dysfunction.

Prior to the 1970s the mortality rate of patients with acute cholangitis was reported to be over $50 \%, 2,3$ but advances in intensive care, new antibiotics, and biliary drainage dramatically reduced the mortality rate to less than $7 \%$ by the 1980 s. ${ }^{4,5}$ However, even in the 1990 s the reported mortality rates in severe cases still ranged from $11 \%$ to $27 \%,{ }^{6-8}$ and even now the severe form of acute cholangitis remains a fatal disease unless appropriate management is instituted.

The clinical diagnosis of acute cholangitis is made on the basis of the clinical findings, such as Charcot's triad, ${ }^{9}$ in combination with the laboratory data and imaging findings, and severity assessment is important because urgent biliary drainage is essential in "severe" cases. However, no standard criteria for the diagnostis and severity assessment of acute cholangitis have ever been established. In this portion of the Tokyo Guidelines, we propose diagnostic criteria and severity assessment criteria for acute cholangitis based on a review of the literature and the consensus of experts reached at the International Consensus Meeting for the Management of Acute Cholecystitis and Cholangitis, held on April 1-2, 2006, in Tokyo.

\section{Diagnostic criteria for acute cholangitis}

A variety of different names and definitions of acute cholangitis are found in the literature, depending on the authors. ${ }^{6,8,10-17}$ Some authors defined acute cholangitis based on clinical sign's such as Charcot's triad (fever and/or chills, abdominal pain, and jaundice), ${ }^{6,16-17}$ while others emphasized the presence of biliary obstruction or the properties of the bile (suppurative cholangitis), ${ }^{10,13-14}$ as a result, there are no standard diagnostic criteria for acute cholangitis. The clinical information used to establish the diagnosis of acute cholangitis includes a history of biliary disease, symptoms and signs, laboratory data, and imaging findings.

\section{Clinical context and manifestations}

A history of biliary disease suggests a clinical diagnosis of cholangitis in patients who present with clinical manifestations such as fever, abdominal pain, and jaundice. Patients with a history of gallstone disease, previous biliary surgery, or the insertion of a biliary stent are more likely to develop biliary infection.
Clinical manifestations are an important factor in making the diagnosis of acute cholangitis. In 1877, ${ }^{9}$ Charcot was the first to describe the clinical triad of fever, jaundice and abdominal pain as a clinical manifestation of acute cholangitis, and in 1959, Reynolds and Dragan $^{18}$ were the first to describe a severe form of cholangitis that included Charcot's triad plus septic shock and mental status change (Reynold's Pentad). Table 1 summarizes the incidence of each clinical manifestation reported in the literature..$^{6,8,10-17}$ Fever and abdominal pain are the most frequently observed clinical manifestations in acute cholangitis, with an incidence of each of up to $80 \%$ or more, whereas jaundice is observed in $60 \%-70 \%$ of cases. The incidence of Charcot's triad is reported in not more than $72 \%$ (range, $15.4 \%$ to $72 \%$ ) of patients with acute cholangitis, and Reynolds' pentad is extremely rare, reported in only $3.5 \%-7.7 \%$ of the patients.

\section{Laboratory data}

Laboratory data indicative of inflammation (e.g., leukocytosis and an elevated C-reactive protein [CRP] level), and evidence of biliary stasis (e.g., hyperbilirubinemia, elevation of biliary enzymes and liver enzymes) are frequently seen in patients with acute cholangitis, and such laboratory findings support the diagnosis. Table 2 summarizes the positive rate for various blood tests in patients with acute cholangitis reported in the literature..$^{5,12,13,17,19-21}$

\section{Imaging findings}

It is usually impossible to identify evidence of bile infection itself by imaging modalities. Imaging evidence of biliary dilatation (evidence of biliary obstruction) and/ or the etiology of the underlying disease (tumor, gallstones, stent-related, etc.) can support the clinical diagnosis of cholangitis.

\section{Diagnostic criteria for acute cholangitis}

Table 3 shows the diagnostic criteria for acute cholangitis that were finally adopted by the Organizing Committee. The basic concepts of the criteria are as follows: (1) Charcot's triad is a definite diagnostic criterion for acute cholangitis, (2) if a patient does not have all the components of Charcot's triad (acute cholangitis is suspected), then definite diagnosis can be achieved if both an "inflammatory response" and "biliary obstruction" are demonstrated by the laboratory data (blood tests) and imaging findings.

\section{Outcome of the Tokyo Consensus Meeting}

More than $90 \%$ of the participants at the Tokyo Consensus Meeting agreed that the four criteria of: (1) a 
Table 1. Incidence of clinical manifestation of acute cholangitis

\begin{tabular}{|c|c|c|c|c|c|c|c|c|c|}
\hline Author & Disease & $n$ & $\begin{array}{l}\text { Charcot's } \\
\text { triad }(\%)\end{array}$ & $\begin{array}{c}\text { Fever } \\
\%\end{array}$ & $\begin{array}{c}\text { Jaundice } \\
\%\end{array}$ & $\begin{array}{l}\text { Abdominal } \\
\text { pain }(\%)\end{array}$ & $\begin{array}{c}\text { Reynold's } \\
\text { pentad (\%) }\end{array}$ & $\begin{array}{c}\text { Shock } \\
\%\end{array}$ & $\begin{array}{c}\text { Disturbed } \\
\text { consciousness }(\%)\end{array}$ \\
\hline Csendes $^{10}$ & ASC & $\begin{array}{r}51 \\
2\end{array}$ & 22 & 38.7 & 65.4 & 92.2 & & 7 & 7.2 \\
\hline Thompson ${ }^{11}$ & $\mathrm{AC}$ & 66 & About 60 & 100 & 66 & 59 & & 7 & 9 \\
\hline Gigot $^{12}$ & $\mathrm{AC}$ & $\begin{array}{r}41 \\
2\end{array}$ & 72 & & & & 3.5 & 7.8 & 7 \\
\hline \multirow[t]{3}{*}{ Boey $^{13}$} & $\mathrm{AC}$ & 99 & 69.7 & 93.9 & 78.8 & 87.9 & 5.1 & 16.2 & 16.2 \\
\hline & $\mathrm{SC}$ & 14 & & & & & 7 & 57 & 28 \\
\hline & NonSC & 72 & & & & & 4 & 8 & 12 \\
\hline \multirow[t]{3}{*}{$\mathrm{O}^{\prime}$ Connor ${ }^{14}$} & $\mathrm{AC}$ & 65 & 60 & & & & 7.7 & 32 & 14 \\
\hline & $\mathrm{SC}$ & 19 & 53 & & & & 5 & 47 & 11 \\
\hline & NonSC & 46 & 63 & & & & 9 & 26 & 15 \\
\hline Lai $^{6}$ & Severe AC & 86 & 56 & 66 & 93 & 90 & & 64 & \\
\hline Haupert $^{15}$ & ASC & 13 & 15.4 & 100 & 61.5 & 100 & 7.7 & 23.1 & 7.7 \\
\hline \multirow[t]{2}{*}{ Welch $^{16}$} & ASC & 5 & 50 & 80 & 60 & & & 0 & 20 \\
\hline & AOSC & 15 & 50 & 88 & 67 & & & 33 & 27 \\
\hline Saharia $^{17}$ & $\mathrm{AC}$ & 78 & & 100 & 61.5 & 100 & & 5.1 & \\
\hline Chijiiwa $^{8}$ & AOSC & 27 & & 63.0 & 70.3 & 96.3 & & 25.9 & 22.2 \\
\hline
\end{tabular}

AC, acute cholangitis; SC, suppurative cholangitis; AOSC, acute obstructive suppurative cholangitis

Table 2. Positive rates for blood tests in acute cholangitis

\begin{tabular}{|c|c|c|c|}
\hline Item & Positive rate $(\%)$ & No. of cases & Author \\
\hline \multirow[t]{3}{*}{$\mathrm{WBC}>10000 / \mathrm{mm}^{3}$} & 79 & 449 & Gigot $^{12}$ \\
\hline & 63 & 78 & Saharia $^{17}$ \\
\hline & 82 & 71 & Boey $^{13}$ \\
\hline \multirow[t]{2}{*}{ Total bilirubin $\uparrow$} & 91 & 78 & Saharia $^{17}$ \\
\hline & 78 & 74 & Boey $^{13}$ \\
\hline \multirow[t]{3}{*}{$\mathrm{ALP} \uparrow$} & 93 & 449 & Gigot $\mathrm{JF}^{5}$ \\
\hline & 92 & 72 & Saharia $^{17}$ \\
\hline & 74 & 74 & Boey $^{13}$ \\
\hline $\mathrm{AST} \uparrow$ & 93 & 45 & Saharia $^{17}$ \\
\hline $\mathrm{ALT} \uparrow$ & 97 & 35 & Saharia $^{17}$ \\
\hline AST or ALT $\uparrow$ & 57 & 74 & Boey $^{13}$ \\
\hline Prolonged prothrombin time & 26 & 74 & Boey $^{13}$ \\
\hline \multirow[t]{2}{*}{ Amylase $\uparrow$} & 7 & 74 & Boey $^{13}$ \\
\hline & 35 & 54 & Boey $^{13}$ \\
\hline Creatinine $\geqq 1.5 \mathrm{mg} / \mathrm{d}$ & 16 & 125 & $\mathrm{Tai}^{5}$ \\
\hline \multirow{2}{*}{ CA19-9 $\uparrow$} & 28 & 25 & $\operatorname{Ker}^{19}$ \\
\hline & 100 & 7 & Albert ${ }^{20}$ \\
\hline Endotoxin $\uparrow$ & 36 & 11 & Kanazawa ${ }^{21}$ \\
\hline
\end{tabular}

Table 3. Diagnostic criteria for acute cholangitis

\begin{tabular}{ll}
\hline A. Clinical context and clinical manifestations & $\begin{array}{l}\text { 1. History of biliary disease } \\
\text { 2. Fever and/or chills } \\
\text { 3. Jaundice }\end{array}$ \\
$\begin{array}{ll}\text { 4. Abdominal pain (RUQ or upper abdominal) } \\
\text { B. Laboratory data }\end{array}$ & $\begin{array}{l}\text { 5. Evidence of inflammatory response } \\
\text { 6. Abnormal liver function tests }\end{array}$ \\
\hline C. Imaging findings & 7. Biliary dilatation, or evidence of an etiology (stricture, stone, stent etc) \\
\hline Suspected diagnosis & Two or more items in A \\
\hline Definite diagnosis & (1) Charcot's triad $(2+3+4)$
\end{tabular}

a Abnormal WBC count, increase of serum CRP level, and other changes indicating inflammation

${ }^{b}$ Increased serum ALP, r-GTP (GGT), AST, and ALT levels 
history of biliary disease, (2) the clinical manifestations, (3) laboratory data indicative of the presence of inflammation and biliary obstruction, and (4) imaging findings indicative of biliary obstruction and/or evidence of etiology were suitable making the diagnosis of acute cholangitis.

\section{Severity assessment of acute cholangitis}

Patients with acute cholangitis may present with anything from a mild, self-limited illness to a severe, potentially life-threatening illness. Most cases respond to initial medical treatment consisting of general supportive therapy and intravenous antibiotics, but some cases do not respond to medical treatment, and the clinical manifestations and laboratory data do not improve. Such cases may progress to sepsis, with or without organ dysfunction, requiring appropriate management that includes intensive care, organ-supportive care, and urgent biliary drainage, in addition to medical treatment.

\section{Severity assessment criteria}

Table 4 summarizes the risk factors reported in the literature for poor outcome in patients with acute cholangitis.2,3,6,10,12,13,15,22-24 Organ dysfunction is the most common predictor of a poor outcome. On the other hand, based on the pathophysiology, "severe" acute cholangitis can also be defined as that which accompanies organ dysfunction caused by sepsis. Thus, "the onset of organ dysfunction" is an important factor in the definition of severe (grade III) acute cholangitis.

Another factor for the severity assessment of acute cholangitis is "response to initial medical treatment"; treatment consisting of general supportive care and antibiotics should be instituted as soon as possible for all patients who are diagnosed with acute cholangitis. Patients diagnosed with acute cholangitis that is not complicated by organ dysfunction, who did not respond to medical treatment and who continue to have SIRS and/or sepsis require additional treatment that includes either a change of antibiotic or biliary drainage. The severity of such cases is classified as moderate (grade II). Patients who respond to medical treatment and whose clinical manifestations and laboratory data improve are classified as having mild (grade I) disease. Table 5 and Table 6 show the concepts and criteria for the severity assessment of acute cholangitis.

Table 4. Prognostic factors in acute cholangitis

\begin{tabular}{lll}
\hline Prognostic factor & \multicolumn{1}{c}{ Positive value } & \multicolumn{1}{c}{ References } \\
\hline Related to organ dysfunction & & \\
$\quad$ Shock & & $2,10,13$ \\
Mental confusion & $>1.5->2.0 \mathrm{mg} / \mathrm{dl}$ & 2,10 \\
Elevated serum creatinine & $>20->64 \mathrm{mg} / \mathrm{dl}$ & $3,10,12,22$ \\
Elevated BUN & $>1.5->2.0 \mathrm{~s}$ & $10,12,24$ \\
Prolonged prothrombin time & $>2.2->10 \mathrm{mg} / \mathrm{dl}$ & 10,23 \\
Hyperbilirubinemia & $<10 \times 10^{4}-<15 \times 10^{4} / \mathrm{mm}^{3}$ & $2,5,6,10,13,22-24$ \\
Reduced platelet count & $>39{ }^{\circ} \mathrm{C}->40^{\circ} \mathrm{C}$ & 3,24 \\
Unrelated to organ dysfunction & $>20000 / \mathrm{mm}^{3}$ & 2,13 \\
High fever & & 2,3 \\
Leukocytosis & & 3,22 \\
Bacteremia & & 3 \\
Endotoxemia & & $6,23,24$ \\
Hypoalbuminemia & & 12 \\
Liver abscess & $>75 \mathrm{mg} / \mathrm{dl}$ & $10,12,15,24$ \\
Medical comorbidity & & $10,12,24$ \\
Elderly patient & & 12,22 \\
Malignancy as etiology & & \\
\hline
\end{tabular}

Table 5. Criteria for severity assessment of acute cholangitis

\begin{tabular}{|c|c|c|c|}
\hline \multirow[b]{2}{*}{ Criterion } & \multicolumn{3}{|c|}{ Severity of acute cholangitis } \\
\hline & $\begin{array}{c}\text { Mild } \\
\text { (grade I) }\end{array}$ & $\begin{array}{l}\text { Moderate } \\
\text { (grade II) }\end{array}$ & $\begin{array}{c}\text { Severe } \\
\text { (grade III) }\end{array}$ \\
\hline Onset of organ dysfunction & No & No & Yes \\
\hline Response to initial medical treatment ${ }^{a}$ & Yes & No & No \\
\hline
\end{tabular}

${ }^{\mathrm{a}}$ Consisting of general supportive care and antibiotics 
Table 6. Definitions of severity assessment criteria for acute cholangitis

Mild (grade I) acute cholangitis

"Mild (grade I)" acute cholangitis is defined as acute cholangitis which responds to the initial medical treatment

Moderate (grade II) acute cholangitis

"Moderate (grade II)" acute cholangitis is defined as acute cholangitis that does not respond to the initial medical

treatment ${ }^{\mathrm{a}}$ and is not accompanied by organ dysfunction

Severe (grade III) acute cholangitis

"Severe (grade III)" acute cholangitis is defined as acute cholangitis that is associated with the onset of dysfunction at least in any one of the following organs/systems:

1. Cardiovascular system Hypotension requiring dopamine $\geqq 5 \mu \mathrm{g} / \mathrm{kg}$ per min, or any dose of dobutamine

2. Nervous system Disturbance of consciousness

3. Respiratory system $\quad \mathrm{PaO} 2 / \mathrm{FiO} 2$ ratio $<300$

4. Kidney Serum creatinine $>2.0 \mathrm{mg} / \mathrm{dl}$

5. Liver PT-INR $>1.5$

6. Hematological system Platelet count $<100000 / \mu \mathrm{l}$

Note: compromised patients, e.g., elderly ( $>75$ years old) and patients with medical comorbidities, should be monitored closely

${ }^{a}$ General supportive care and antibiotics

\section{Outcome of the Tokyo Consensus Meeting}

More than $70 \%$ of the participants at the Tokyo Consensus Meeting agreed that the severity of acute cholangitis should be divided into three grades mild (grade I), moderate (grade II), and severe (grade III). To stratify acute cholangitis into the three grades, two different criteria were necessary, and it was decided to use "onset of organ dysfunction" and "response to the initial medical treatment" as criteria for the severity assessment of acute cholangitis (Table 5).

Acknowledgments. We would like to express our deep gratitude to the Japanese Society for Abdominal Emergency Medicine, the Japan Biliary Association, and the Japanese Society of Hepato-Biliary-Pancreatic Surgery, who provided us with great support and guidance in the preparation of the Guidelines. This process was conducted as part of the Project on the Preparation and Diffusion of Guidelines for the Management of Acute Cholangitis (H-15-Medicine-30), with a research subsidy for fiscal 2003 and 2004 (Integrated Research Project for Assessing Medical Technology), sponsored by the Japanese Ministry of Health, Labour, and Welfare.

We also truly appreciate the panelists who cooperated with and contributed significantly to the International Consensus Meeting, held in Tokyo on April 1 and 2, 2006.

\section{References}

1. Lipsett PA, Pitt HA. Acute cholangitis. Surg Clin North Am 1990;70:1297-312. (level 4)

2. Andrew DJ, Johnson SE. Acute suppurative cholangitis, a medical and surgical emergency. Am J Gastroenterol 1970;54:141-54. (level 4)
3. Shimada H, Nakagawara G, Kobayashi M, Tsuchiya S, Kudo T, Morita S. Pathogenesis and clinical features of acute cholangitis accompanied by shock. Jpn J Surg 1984;14:269-77. (level 4)

4. Thompson JE Jr, Pitt HA, Doty JE, Coleman J, Irving C. Broad spectrum penicillin as an adequate therapy for acute cholangitis. Surg Gynecol Obstet 1990;171:275-82. (level 4)

5. Tai DI, Shen FH, Liaw YF. Abnormal pre-drainage serum creatinine as a prognostic indicator in acute cholangitis. Hepatogastroenterology 1992;39:47-50. (level 4)

6. Lai EC, Tam PC, Paterson IA, Ng MM, Fan ST, Choi TK, et al. Emergency surgery for severe acute cholangitis. The high-risk patients. Ann Surg 1990;211:55-9. (level 4)

7. Liu TJ. Acute biliary septic shock. HPB Surg 1990;2:177-83. (level 4)

8. Chijiiwa K, Kozaki N, Naito T, Kameoka N, Tanaka M. Treatment of choice for choledocholithiasis in patients with acute obstructive suppurative cholangitis and liver cirrhosis. Am J Surg 1995;170:356-60. (level 2b)

9. Charcot M. De la fievre hepatique symptomatique. Comparaison avec la fievre uroseptique. Lecons sur les maladies du foie des voies biliares et des reins. Paris: Bourneville et Sevestre; 1877. p. 176-85.

10. Csendes A, Diaz JC, Burdiles P, Maluenda F, Morales E. Risk factors and classification of acute suppurative cholangitis. $\mathrm{Br} \mathrm{J}$ Surg 1992;79:655-8. (level 2b)

11. Thompson JE Jr, Tompkins RK, Longmire WP Jr. Factors in management of acute cholangitis. Ann Surg 1982;195:137-45. (level 4)

12. Gigot JF, Leese T, Dereme T, Coutinho J, Castaing D, Bismuth $\mathrm{H}$. Acute cholangitis. Multivariate analysis of risk factors. Ann Surg 1989;209:435-8. (level 4)

13. Boey JH, Way LW. Acute cholangitis. Ann Surg 1980;191:264-70. (level 4)

14. O'Connor MJ, Schwartz ML, McQuarrie DG, Sumer HW. Acute bacterial cholangitis: an analysis of clinical manifestation. Arch Surg 1982;117:437-41. (level 4)

15. Haupert AP, Carey LC, Evans WE, Ellison EH. Acute suppurative cholangitis. Experience with 15 consecutive cases. Arch Surg 1967;94:460-8. (level 4)

16. Welch JP, Donaldson GA. The urgency of diagnosis and surgical treatment of acute suppurative cholangitis. Am J Surg 1976;131: 527-32. (level 4)

17. Saharia PC, Cameron JL. Clinical management of acute cholangitis. Surg Gynecol Obstet 1976;142:369-72. (level 4)

18. Reynolds BM, Dragan EL. Acute obstructive cholangitis. A distinct syndrome. Ann Surg 1959;150:299-303. 
19. Ker CG, Chen JS, Lee KT, Sheen PC, Wu CC. Assessment of serum and bile levels of CA19-9 and CA125 in cholangitis and bile duct carcinoma. J Gastroenterol Hepatol 1991;6:505-8. (level 4)

20. Albert MB, Steinberg WM, Henry JP. Elevated serum levels of tumor marker CA19-9 in acute cholangitis. Dig Dis Sci 1988;33:1223-5. (level 4)

21. Kanazawa A, Kinoshita H, Hirohashi K, Kubo S, Tsukamoto T, Hamba $\mathrm{H}$, et al. Concentrations of bile and serum endotoxin and serum cytokines after biliary drainage for acute cholangitis. Osaka City Med J 1997;43:15-27. (level 4)

22. Thompson J, Bennion RS, Patt HA. An analysis of infectious failures in acute cholangitis. HPB Surgery 1994;8:139-44. (level 4)

23. Hui CK, Lai KC, Yuen MF, Ng M, Lai CL, Lam SK. Acute cholangitis - predictive factors for emergency ERCP. Aliment Pharmacol Ther 2001;15:1633-7. (level 4)

24. Hanau LH, Steigbigel NH. Acute (ascending) cholangitis. Infectious Dis Clin North Am 2000;14:521-46. (level 4)

\section{Discussion at the Tokyo Consensus Meeting}

\section{Diagnostic criteria for acute cholangitis}

"Acute cholangitis" is a clinical diagnosis. A definite diagnosis cannot be made on the basis of the results of any single test. The diagnosis of acute cholangitis is made on the basis of: (1) a history of biliary disease, (2) the clinical manifestations, (3) laboratory data that indicate the presence of inflammation and biliary obstruction, and (4) imaging findings that indicate biliary obstruction. More than $90 \%$ of participants at the International Consensus Meeting agreed that these four criteria were suitable for making the diagnosis of acute cholangitis (consensus was reached).

In terms of the clinical context and manifestations, a history of biliary disease and the clinical presentation are important factors in reaching the diagnosis. A history of biliary disease, such as gallstones, a history of previous biliary surgery, and having an indwelling biliary stent play an important role in making the diagnosis, as agreed upon by many participants at the Consensus Meeting. The more important clinical manifestations are clinical signs, such as Charcot's triad (fever and/or chills, abdominal pain, and jaundice). According to the literature, $50 \%-70 \%$ of acute cholangitis patients present with Charcot's triad, meaning that more than onethird of acute cholangitis patients do not present with all the components of Charcot's triad. The laboratory data and imaging findings can provide evidence to support the diagnosis in patients who have clinical manifestations of acute cholangitis but who do not show all the components of Charcot's triad (refer to Table 3).

\section{Severity assessment criteria for acute cholangitis}

A systematic review of the literature revealed that there were no standard criteria for either the diagnosis or se- verity assessment of acute cholangitis. Some authors have defined acute cholangitis associated with Reynold's pentad (Charcot's triad plus "shock" and "disturbance of consciousness") or organ dysfunction as "severe", while others have referred to it as "toxic cholangitis" or "acute obstructive suppurative cholangitis (AOSC)". A proposal that the onset of dysfunction of at least one organ be used as the criterion for severe (grade III) disease was supported by more than $90 \%$ of the panelists at the International Consensus Meeting (consensus was reached).

There was some argument about whether the score on an acute physiology scoring system, such as Acute physiology and chronic health evaluation (APACHE II) score or a multiple organ dysfunction scoring system, such as Marshall's system, or sepsis-related organ failure assessment (SOFA) system should be used as a criterion for severe (grade III) acute cholangitis. The principal advantage of these scoring systems is that they provide gradations of severity. The APACHE II system has been validated, especially for critical care patients, including patients with sepsis, and acute cholangitis can be interpreted as a subset of sepsis. The disadvantage of these scoring systems is that the scores are sometimes troublesome to calculate, and critically speaking, they have not been satisfactorily validated in patients with acute cholangitis. The vote on this argument showed that $37.8 \%$ of the panelists supported the use of APACHE II and $62.2 \%$ did not. As a result of this vote, the chairmen of this session, Drs. Yoshifumi Kawarada (Japan) and Henry Pitt (USA), proposed to remit the final decision on whether or not APACHE II should be included as a criterion for severe (grade III) acute cholangitis to the Organizing Committee, and this proposal was approved by the audience.

After the meeting, the Organizing Committee decided not to include the use of the APACHE II score as a criterion for the definition of severe (grade III) acute cholangitis, and we established the criteria by evaluating the presence or absence of the dysfunctions of six major organs/systems (refer to Table 6).

Deciding on the criteria for the assessment of acute cholangitis as moderate was the hardest part of this session. More than $70 \%$ of the participants agreed that a middle category of severity - moderate (grade II) was necessary for acute cholangitis (consensus was reached).

The original definition of moderate (grade II) acute cholangitis was "acute cholangitis that requires biliary drainage but is not complicated by organ dysfunction." However, more than $80 \%$ of the participants voted against the need for biliary drainage as a criterion because it is a therapeutic intervention that should be selected only after the severity assessment has been completed. Thus, another criterion was needed in order 
to stratify acute cholangitis into three grades. Other criteria for assessing acute cholangitis as moderate (grade II) were suggested by the audience. The most accepted criterion during the discussion was "resistance to initial treatment", with some others being "recurrence of symptoms" and "SIRS". The chairmen of this session also proposed to remit the final decision to the Organizing Committee, and this proposal was approved by the audience.

After the Meeting, the Organizing Committee concluded that the criterion for assorting into moderate (grade II) and mild (grade I) acute cholangitis should be "response to initial medical treatment consisting of general supportive care (intravenous fluid) and antibiotics," i.e., acute cholangitis that responds to medical treatment is defined as mild (grade I) acute cholangitis, whereas acute cholangitis that does not respond to the initial medical treatment but does not have organ dysfunction is defined as moderate (grade II) acute cholangitis (refer to Tables 5 and 6). No specific data or findings were adopted as criteria, because it is impossible to predict the need for biliary drainage based on the laboratory data or other findings. It was therefore concluded that we considered that it is important to stratify acute cholangitis as "severe" or "non-severe" at the time of diagnosis. Patients with the former require urgent biliary drainage in addition to general and organsupportive treatment, while patients with the latter should be monitored to determine whether they respond to the initial medical treatment. 